\section{Immuntherapie: Erfolge mit sublingualer Applikation}

\section{Die lokale Applikation von Allergoiden führt zu einer klinischen Besserung der milbeninduzierten Rhinokonjunktivitis.}

$\mathrm{D}$ ie häufigen subkutanen Injektionen machen die spezifische Immuntherapie bei vielen Patienten unbeliebt. Ein Ausweg wäre die topische Applikation der Allergene auf die Schleimhäute. Es ist zwar bekannt, daß die Allergene von dort resorbiert werden und in die lokalen Lymphknoten gelangen. $\mathrm{Ob}$ sie aber auch einen Shift von der TH2- zur TH1-Antwort auslösen und ob dies dann tatsächlich zu einer spürbaren Verbesserung der allergischen Diathese führt, war bislang unbewiesen.

Italienische Forscher führten hierzu nun die erste randomisierte plazebokontrollierte Studie durch: Eingeschlossen waren 20 Patienten mit saisonaler allergischer Rhinokonjunktivitis und nachgewiesener Sensibilisierung auf Hausstaubmilbe. 6 Patienten litten überdies an mildem Asthma. Sie erhielten Tabletten, die entweder Plazebo oder ein monomeres Allergoid von Dermatophagoides pteronyssinus und $\mathrm{D}$. farinae enthielten.

Die Patienten ließen die Tabletten während ein bis zwei Minuten unter der Zunge zergehen und schluckten sie dann hinunter. Die Allergoiddosis wurde in der Aufbauphase von $25 \mathrm{AU}$ auf 1000 AU gesteigert. Während der Erhaltungsphase betrug sie $2000 \mathrm{AU}$, was dem 20fachen der Dosis bei subkutaner Injektion entspricht. Die Einnahme von Steroiden, Antihistaminika und Betamimetika war erlaubt. Die Patienten führten ein Symptomtagebuch. Außerdem wurde im Sommer vor Studienbeginn sowie nach 12 und 24 Monaten ein Abstrich der Konjunktiva nach Allergenprovokation auf entzündliche Zellen untersucht.

Die Patienten der Verumgruppe berichteten über eine konstante und im Vergleich zur Plazebogruppe signifikante Besserung der rhinokonjunktivalen Beschwerden während der Wintermonate. Im Sommer waren die Be- schwerden in beiden Gruppen mild. Die konjunktivale Reaktion auf Allergenprovokation war jedoch unter Verum deutlich abgeschwächt. Die entzündliche Infiltration und die Expression von ICAM-1 in den Epithelien war bereits im ersten Jahr signifikant geringer als unter Plazebo. Auch die Serumkonzentrationen des eosinophilen kationischen Proteins waren signifikant verringert. - Als einzige Neben- riner iner der neueren Vertreter inhalierbarer Steroide, das Fluticason, hatte sich in vitro potenter als einige der älteren Kortikoide erwiesen. Nachdem es darüber hinaus eine vergleichsweise äußerst geringe orale systemische Verfügbarkeit aufweist, lag es nahe, hier einen möglicherweise höheren therapeutischen Index $\mathrm{zu}$ vermuten als bei älteren Substanzen.

In zwei Metaanalysen wollte man hierüber Klarheit gewinnen. Ausgewertet wurden 7 Studien, in denen Wirksamkeit und Sicherheit von Fluticason gegenüber Budesonid untersucht wurden, sowie 7 Vergleichsstudien mit Beclometason. Kriterien für die Aufnahme einer Studie war, daß Fluticason darin halb so hoch wie die Vergleichssubstanz dosiert wurde und daß man den morgendlichen Peak-flowWert zur Evaluation des Therapieerfolgs bestimmt hatte. Zur Feststellung einer systemischen Wirkung wurden die morgendlichen Serumkonzentrationen von Kortisol gemessen. wirkung trat bei einem Patienten oraler ,Juckreiz“ auf, der sich nach wenigen Minuten spontan zurückbildete.

Im Editorial betont P.G. Holt vom Institute for Child Health Research in West Perth, Australien, die Stärken der Studie: Die lange Nachbeobachtungszeit von zwei Jahren sowie der Einschluß objektiver Parameter. Einzige Schwäche sei die geringe Teilnehmerzahl. Allerdings führten bis auf einen alle Patienten die Therapie bis zum Ende durch. Studien mit größeren Patientenzahlen müssen nun die Effektivität einer sublingualen Allergentherapie bestätigen.

(rme)

Quelle: Passalacqua G et al.: Randomised controlled trial of local allergoid immunotherapy on allergic inflammation in mite-induced rhinoconjunctivitis. Lancet (1998); 351:629-632. Holt PG et al.: Sublingual immunotherapy for allergic respiratory disease. Lancet (1998); 351:613-614.

\title{
Inhalative Steroide: Unterschiede in der Nutzen-Risiko-Relation?
}

\section{Fluticason scheint besonders in höherer Dosierung eine günstigere Nutzen-Risiko-Relation als ältere Steroide zu haben.}

Die Metaanalyse zeigte eine eindeutig effektivere Behandlung durch Fluticason gegenüber Budesonid. In niedrigen Dosen fand sich in bezug auf die Therapiesicherheit praktisch kein Unterschied zwischen den Steroiden. Dagegen fiel der Vergleich signifikant zugunsten Fluticason aus, wenn man höhere Konzentrationen (Budesonid $\geq 1200 \mu \mathrm{g}$ pro Tag resp. Fluticason $\geq$ $500 \mu \mathrm{g}$ ) betrachtete; auch die gepoolte Analyse ergab hier eindeutige Differenzen bei den Kortisolwerten.

Im Vergleich mit Beclometason zog Fluticason bezüglich des Therapieeffektes (Peak-flow) gleich. Auch Beclometason unterdrückte in Dosen über $1000 \mu \mathrm{g}$ die endogene Kortisolsekretion stärker als Fluticason in klinisch äquivalenter Dosis $(500 \mu \mathrm{g})$, die gepoolte Analyse ergab hier allerdings keine signifikante Differenz. (wpa)

Barnes NC et al.: Clinical experience with fluticasone propionate in asthma: a meta-analysis of efficacy and systemic activity compared with budesonide and beclomethasone dipropionate at half the microgram dose or less. Respir Med (1998); 92:95-104. 